Research Paper

\title{
Neoadjuvant Chemoradiation Treatment for Resectable Esophago-Gastric Cancer: A Systematic Review and Meta-Analysis
}

\author{
Xiangyu Meng1, Lu Wang², Yan Zhao ${ }^{\bowtie}$, Bo Zhu³, Ting Sun³, Tao Zhang1, Xiaohu Gu' ${ }^{1}$, Zhichao Zheng1 \\ 1. Department of Gastric Surgery, Cancer Hospital of China Medical University/Liaoning Cancer Hospital, Shenyang, Liaoning, China \\ 2. Department of Ultrasonography, the Fourth Affiliated Hospital of China Medical University, Shenyang, Liaoning, China \\ 3. Department of Information Management, the Information Center, Cancer Hospital of China Medical University/Liaoning Cancer Hospital, Shenyang, \\ Liaoning, China \\ $\square$ Corresponding author: Yan Zhao, Department of Gastric Surgery, Cancer Hospital of China Medical University/Liaoning Cancer Hospital, No.44 Xiaoheyan \\ Road, Dadong District, Shenyang 110042, Liaoning, China. E-mail:dr.zhaoyan@126.com; Tel: (024)31916622 \\ (C) Ivyspring International Publisher. This is an open access article distributed under the terms of the Creative Commons Attribution (CC BY-NC) license \\ (https://creativecommons.org/licenses/by-nc/4.0/). See http://ivyspring.com/terms for full terms and conditions.
}

Received: 2018.03.06; Accepted: 2018.10.27; Published: 2019.01.01

\begin{abstract}
Background: Neoadjuvant chemoradiation (CRT) remains controversial in the treatment of the oesophagus or gastro-oesophageal junction (GOJ) carcinomas.

Methods: We conducted a meta-analysis to assess the efficacy and safety of Neoadjuvant CRT plus surgery comparing with neoadjuvant CT plus surgery or surgery alone. Feasible studies were searched from electronic databases. The outcomes of survival, RO resection rate and adverse effects were analyzed. The outcomes were measured with relative risk (RR) and odds ratio(OR).

Results: Seventeen records including 4095 patients were included. Neoadjuvant CRT improved 1-,2-,3-and 5-year survival. The relative risk (RR) [95\% confidence interval $(\mathrm{Cl}), \mathrm{P}$ value] was respectively $1.08(1.03-1.14,0.002), 1.21(1.12-1.32,<0.00001), 1.31(1.09-1.58,0.004), 1.38(1.17-1.62$, $<0.001)$.In subgroup analysis, patients with squamous cell carcinoma benefited more survival advantage from neoadjuvant CRT than those with adenocarcinoma[1.23(1.15-1.33)vs1.11 (1.03-1.19)]. A significant advantage was observed in analysis of neoadjuvant CRT for PFS [1.32 $(1.22-1.44),<0.00001]$. Tests for DFS between neoadjuvant CRT and neoadjuvant CT or surgery alone were not statistically significant $[1.06(0.97-1.17,0.19)]$. Neoadjuvant CRT was associated with higher R0 resection [2.58(1.75-3.82), $<0.00001]$ and $P C R$ rate $[4.37(2.68-7.13),<0.00001]$. Neoadjuvant CRT lowered the local recurrence rate $[0.52(0.39-0.69),<0.00001]$ and didn't control distant metastasis rate[0.85(0.67-1.08),0.19]. There was no evidence that neoadjuvant CRT increased the treatment-related mortality [1.27(0.95-1.71),0.11]. Neoadjuvant CRT plus surgery did not increase the risk of adverse events morbidity[1.14(0.99-1.32),0.08].

Conclusion: Patients with oesophagus or GOJ carcinomas can obtain a survival advantage from neoadjuvant CRT. The addition of radiation was efficacy and safe in range. However, these results need further high-quality prospective RCTs confirmation.
\end{abstract}

Key words: oesophagus or gastro-oesophageal junction (GOJ) carcinomas, neoadjuvant chemoradiation, survival, meta-analysis

\section{Introduction}

Cancers of the oesophagus or gastrooesophageal junction (GOJ) are often locally advanced or metastatic and have an unsatisfactory prognosis[1]. Noticeably adenocarcinoma (ADC) now accounts for more than $50 \%$ of newly diagnosed cases[2]. Nowadays, surgical therapy is still the nucleus of treatment for the oesophagus or GOJ carcinoma; However, despite progress in surgical techniques and 
the extension of surgical resection, survival has not improved. The 5-year survival rates range from $15 \%$ to $30 \%$, which indicates the urgent need for additional therapies[3]. As we all know, neoadjuvant CT for advanced esophageal and gastric cancer have been accepted in various prospective randomized studies and meta-analyses due to the beneficial on survival over last decade. The notable MAGIC and FFCD trials, both of which included rather plenty of patients and were scientifically statistical, showed a significant benefit for neoadjuvant CT.

Neoadjuvant CRT is a new subject drawing great attention. The utilization of radiotherapy in the treatment of advanced gastric and lower esophageal cancer was not only sterilizing cancer cells that might dislodge and seed during surgery, but also made unresectable tumors shrink sufficiently to allow resection[4]. However, pathological changes include edematous, thickened and hyperemic mucosal manifestation in the early stage from radiotherapy also added the negative effect on survival[5]. Although the RCT of preoperative CRT for esophageal cancer in 1992 by Nygaard et al reported radiotherapy combined with CT could prolong survival[6]. Nevertheless there were still many phase III trials and meta-analyses comparing between CRT by surgery and surgery alone not showing a benefit for neoadjuvant CRT. The patients with major pathologic response benefited from a significant improvement in OS compared to no response or minor pathologic changes after neoadjuvant therapy in gastro-esophageal cancers[33,34]. Meanwhile, Piro et al thought that the unsatisfactory result in OS of the different therapeutic approaches might be partially ascribed to the incorrect application in patients without discriminating potential responders by identifying their biomarkers[35].The contradictory results above made neoadjuvant CRT be considered only for selected patients. Whether neoadjuvant chemoradiation can improve patient survival remains controversial.

Therefore we used meta-analysis to analyze the results of published randomized trials and deeply discuss the role of neoadjuvant CRT followed by surgery for the oesophagus or GOJ cancers on survival effect as compared with neoadjuvant CT followed by surgery or surgery alone. Thus, the promising strategy in the treatment of the oesophagus or GOJ cancers would be identified in details and it may be used as reference for selection of clinical treatment.

\section{Methods}

\section{Literature search}

Studies published up to August 7, 2018 were searched from PubMed, Embase, Web of science, the Cochrane Library, the Cochrane Controlled Trials Register, WanFan data, VIP database and China National Knowledge Infrastructure(CNKI) using the following search term: "oesophageal/ esophageal/ esophagus/ gastroesophageal junction/ esophagogastric junction/gastric cancer", "preoperative or neoadjuvant chemoradiotherapy/ radiochemotherapy", "randomized controlled trials(RCT)". There were no language restrictions. In addition, related studies and potentially relevant articles were also searched. References of the included articles were searched by hand.

\section{Inclusion criteria and exclusion criteria}

The studies that met the following inclusion criteria were selected: 1.published RCT studies. 2.clear statement in the Materials and Methods section. 3. eligible patients were randomly assigned to treatment and control arms. 4 . the treatment arm: preoperative CRT plus surgery; the control arm: surgery alone or preoperative CT plus surgery. 5. included patients with resectable, pathologic diagnosis carcinoma of the esophagus, gastro-esophageal junction or stomach. 6. included studies with a low risk of selection, performance ,detection, attrition ,reporting and other bias. Non-RCTs, case reports, reviews, conference presentation and fundamental researches were excluded.

\section{Data extraction and quality assessment}

Three authors (M.XY,S.T,Z.Y) independently selected the trials and extracted data according to the criteria. The following data were collected: author information, year, country, sample size, treatment approach and schedule, median follow-up, R0 resection rate, pathological reaction, overall survival(OS), disease-free survival(DFS), progression-free survival (PFS), metastasis and recurrence rate, mortality and morbidity. Disagreements were resolved by discussion. The OS, DFS, PFS were assessed as the primary outcomes. The secondary outcomes were R0 resection rate, pathological reaction, metastasis and recurrence rate, perioperative mortality and morbidity. Two authors (Z.B, Z.T) independently conducted the quality assessment based on the Newcastle-Ottawa Scale (NOS) which is a semi quantitative method for assessing the quality of studies, and consisted of three main parts: selection (4 points), comparability (2 points) and outcome (3 points). The quality of study was determined on a scale from zero to nine points. Studies with seven or more points were regarded as "high quality", studies with the points from four to six were regard as "moderate quality", and otherwise, the study was regarded as "low quality". The above were done in duplicate with disagreements handled 
by discussion to reach a consensus.

\section{Statistical analysis}

Data analysis was conducted using the Review Manager 5.3.0. Meta-analyses on overall survival were measured with relative risk (RR), and R0 resection rate, metastasis and recurrence rate, perioperative mortality and morbidity were performed using odds ratios (OR) with its 95\% confidence interval (CI) as pooled effect measures. Intention-to-treat (ITT) analyses were conducted in most evaluation. Heterogeneity was assessed using I 2 statistics. When $\mathrm{I} 2<50 \%$ and $\mathrm{P}>0.1$, the fixed model was conducted; Otherwise, the Mantel-Haenszel (M-H) random model was selected and subgroup analysis or sensitivity analysis were performed. Statistical significance was set at $\mathrm{P} \leq 0.05$. Forest plots were used to show the results visually and to assess bias.

\section{Results}

\section{Study Characteristics}

We collected a total of 2497 records and excluded 240 duplicates from the initial search. After screening titles and abstracts of 2269 records, the full texts of 187 records were assessed on inclusion and exclusion criteria. 17 records [6-22] were eventually eligible for the meta-analysis, including 4095 patients (Figure 1).

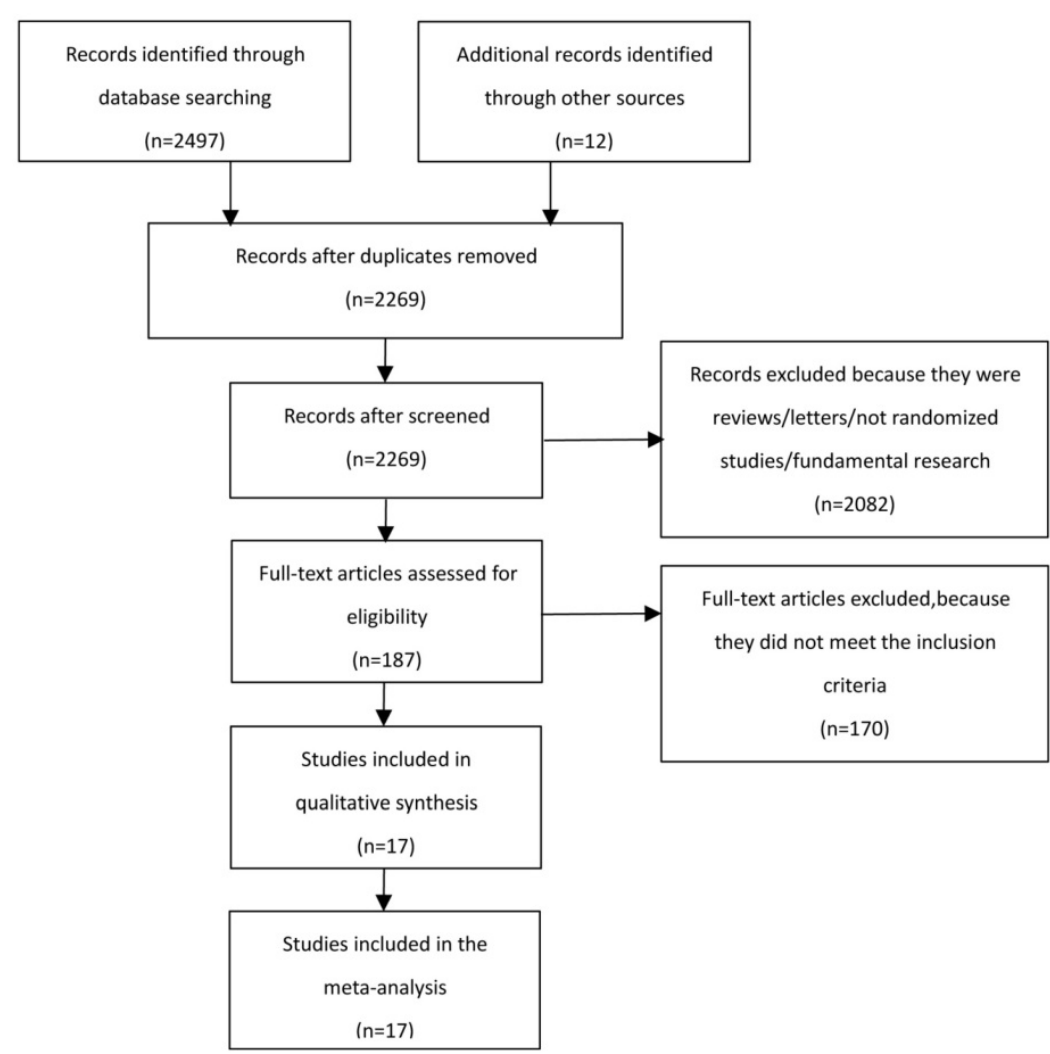

Figure 1. Flow chart of the literature search
Among 17 records, there were 2 records $[6,10]$ including 3 study arms, being neoadjuvant CRT plus surgery, neoadjuvant CT plus surgery and surgery alone. So we separately conducted meta-analysis on inclusion criteria. 13 records [6-8, 10-14, 18-22] compared neoadjuvant $\mathrm{CT}$ plus surgery with surgery alone. 6 records $[6,9-10,15-17]$ were in regard to compare neoadjuvant CRT plus surgery with neoadjuvant CT plus surgery. 17 records included eleven countries and were published from 1992 to 2017. 5 records[6-7, 11-13] reported the oesophagus position. 3 records $[16-17,22]$ reported the GOJ position. 7 records $[9-10,14-15,19-21]$ were restricted to the oesophagus and GOJ positions. Record of oesophagus/GOJ/stomach positions was only one [18]. 5 records[15-17, 21-22] researched adenocarcinoma. 4 records $[6,7,11,13]$ researched squamous cell carcinoma, and the remaining records enrolled both adenocarcinoma and squamous cell carcinoma. The main information of 17 records was listed in table 1. The quality score ranged from 5 to 8.4 records were evaluated as 8 scores, 6 records were evaluated as 7 scores, 4 records were evaluated as 6 scores, 3 records were evaluated as 5 scores. All the included records were regarded as moderate and high quality. The characteristic and quality of the included studies is shown in Table S1.

\section{Primary outcomes}

Meta-analysis about OS, DFS and PFS were including 1-year survival, 2-year survival, 3-year survival and 5-year survival. For OS, a total of 14 records [6-9, 11-12, 14-21] were referred to. 14 records $[6-9,11-12,14-21]$ reported 1-year survival. 11 records $[6-9,12,14-17$, 20-21] reported 2-year survival. 14 records [6-9, 11-12, 14-21] reported 3-year survival. 12 records $[7-8,12-21]$ reported 5-year survival. The effect of neoadjuvant CRT plus surgery on survival was associated with a significant increase in 1-year survival, 2-year survival, 3-year survival and 5-year survival when compared to neoadjuvant CT plus surgery or surgery alone. The RR(95\% CI, P value) was separately 1.08 $(1.03-1.14, \quad 0.002), \quad 1.21 \quad(1.12-1.32$, $<0.00001), \quad 1.31 \quad(1.09-1.58,0.004), 1.38$ (1.17-1.62, <0.001). The pooled RR of neoadjuvant CRT plus surgery was 1.18 (1.14-1.23, <0.00001) compared to neoadjuvant CT plus surgery or surgery alone. The heterogeneity test was not significant $(\mathrm{I} 2=48 \%)$, the fixed model was 
used (Table 2). However, the 14 records contained adenocarcinoma or squamous cell carcinoma and reported different sites. So the subgroups classified by pathogenic type and tumor site were separately calculated for OS. In adenocarcinma subgroup, 9 records [8-10, 14-17, 20-21] reported 1-,2-,3-year survival and 8 records[8, 10,14-17, 20-21] reported 5 -year survival. In squamous cell carcinoma subgroup, 8 records[6-11, 14, 20] reported 1-,3-year survival, 7 records[6-10, 14, 20] reported 2-year survival and 6 records[7-9, 13-14, 20] reported 5-year survival. Although there was no statistically significant in 1-,2-,3- and 5-year survival between two groups, the pooled RR of neoadjuvant CRT plus surgery was $1.11(1.03-1.19,0.004)$ in adenocarcinma subgroup. Oppositely, statistically significances for $1-, 2-, 3-$ and 5-year survival in squamous cell carcinoma were observed between neoadjuvant CRT plus surgery and neoadjuvant CT plus surgery or surgery alone(a pooled RR $1.23,95 \%$ CI 1.15-1.33, $\mathrm{P}<0.00001)$ (Table 3). A heterogeneity was tested to adjust to use a random model $(\mathrm{I} 2=52 \%)$ in adenocarcinma subgroup. The subgroup-analysis was further performed to evaluate the sources of the heterogeneity. Unfortunately, there were still no statistically significances between whether neoadjuvant CRT plus surgery was compared with neoadjuvant CT plus surgery or surgery alone (Table S2). We also conducted a meta-analysis for different tumor sites. On the other hand, there were 6 related-records reporting survival rate of patients with esophagus cancer in esophagus subgroup and 2 recoreds refer to survival rate of patients with GOJ cancer were reported. In esophagus subgroup, no significant difference was observed at 1-,2-,3- and 5 -year survival, but a trend to wards improved survival in favour of CRT were detected at 1-,2-,3- and 5 -year survival. The pooled RR ( $95 \% \mathrm{CI}, \mathrm{P}$ value) was 1.06 (0.98-1.14,0.14)(Table 3).Significant benefits were shown at 2- and 3-year survival in GOJ subgroup, but no survival differences were observed at 1- and 5-year survival in GOJ subgroup. The pooled RR $(95 \% \mathrm{CI}, \mathrm{P}$ value) was $1.33(1.15-1.53,<0.0001)$ (Table 3$)$.For the records reporting DFS $(\mathrm{n}=3)[7,12,15]$ and PFS $(n=4)[8,9,14,17]$, the results of heterogeneity tests (I2,P )were respectively $(0 \%, 0.45)$ and $(19 \%, 0.25)$. Therefore, the fixed models were used. The metaanalysis yielded RRs(95\%CI, P value) of 1.13 (1.00$1.28,0.05), 1.08(0.90-1.29,0.39), 0.99(0.78-1.26,0.94)$ and $0.91(0.64-1.30,0.62)$ for neoadjuvant CRT plus surgery compared to neoadjuvant CT plus surgery or surgery alone in 1-,2-,3-and 5-year DFS. The pooled RR (95\% CI, P value) for DFS was 1.06 (0.97-1.17, 0.19), indicating that there was no significant DFS benefit. However, the RRs (95\% CI, P value) of $1-, 2-, 3$-and 5-year PFS were separately 1.23 (1.09-1.39, 0.0006), 1.39 (1.18-1.65, <0.0001), 1.26 (0.96-1.66, 0.09), $1.53(1.20-1.95,0.0004)$. The pooled $\mathrm{RR}(95 \% \mathrm{CI}, \mathrm{P}$ value $)$ for PFS was $1.32(1.22-1.44,<0.00001)$. The data showed that there was a significant PFS benefit in 1-,2-,5-year survival for neoadjuvant CRT plus surgery compared to neoadjuvant CT plus surgery or surgery alone (Table 4).

\section{Secondary outcomes}

\section{$\mathrm{R} 0$ resection and pathological complete response rate} (PCR)

14 records[6-12, 15-22] reported the $\mathrm{R} 0$ resection and meta-analysis indicated that a statistically significant difference(OR 2.58, 95\% CI 1.75-3.82, $\mathrm{P}<0.00001$ ) (Figure 2A). As an obvious heterogeneity was observed(I2=57\%, $\mathrm{P}=0.004)$, we used random model to calculate the pooled OR and performed subgroup analysis, indicating a significant difference between neoadjuvant CRT plus surgery and neoadjuvant CT plus surgery(OR 2.11, 95\% CI 1.15-3.86, $\mathrm{P}=0.02$ ) or surgery alone (OR 2.96, 95\% CI 1.93-4.55, $\mathrm{P}<0.00001$ )(Figure S1). 5 records[9-11,16-17] reported pCR and the meta-analysis yielded the pooled OR(95\%CI, P value) of $4.37(2.68-7.13,<0.00001)$ for neoadjuvant CRT plus surgery compared to neoadjuvant CT plus surgery or surgery alone(Figure 2B).

\section{Metastasis and recurrence rate}

9 records[8-9, 11-14, 18-19] reported the local recurrence rate and 8 records $[8,11-14,17-19]$ reported the distant metastasis rate. There were no heterogeneity to be observed in these two outcomes ( $\mathrm{I} 2=0 \%, \mathrm{P}=0.72 ; \mathrm{I} 2=31 \%, \mathrm{P}=0.18)$. The fixed models were used to calculate two pooled ORs respectively. Obviously, the patients by treated by neoadjuvant CRT plus surgery had a lower incidence of local recurrence compared to neoadjuvant $\mathrm{CT}$ plus surgery or surgery alone (OR 0.52, 95\% CI 0.39-0.69, $\mathrm{P}<0.00001)$, but no significant difference between two arms was shown in the distant metastasis (OR 0.85, 95\% CI 0.67-1.08, $\mathrm{P}=0.19$ )(Figure 2C,D).

Table 1. Basic characteristics of studies included

\begin{tabular}{lllllll}
\hline $\begin{array}{l}\text { Study and } \\
\text { year }\end{array}$ & Country & Cancer position/histology(\%) Sample size & $\begin{array}{l}\text { Treatment } \\
\text { approach }\end{array}$ & $\begin{array}{l}\text { Median } \\
\text { follow-up (m) }\end{array}$ & Treatment Schedule & $\begin{array}{l}\text { NOS } \\
\text { score }\end{array}$ \\
\hline $\begin{array}{l}\text { CRT-S V.S } \\
\text { Bosset, } 1997\end{array}$ & France & Oesophageal & $(282) 143$ V.139 & CRT-S V.S & 55.2 & CRT:37Gy/3.7Gy-18.5f/2w; \\
\hline
\end{tabular}




\begin{tabular}{|c|c|c|c|c|c|c|c|}
\hline $\begin{array}{l}\text { Study and } \\
\text { year }\end{array}$ & Country & Cancer position/ histology(\%) & Sample size & $\begin{array}{l}\text { Treatment } \\
\text { approach }\end{array}$ & $\begin{array}{l}\text { Median } \\
\text { follow-up (m) }\end{array}$ & Treatment Schedule & $\begin{array}{l}\text { NOS } \\
\text { score }\end{array}$ \\
\hline & & SCC & & & & $\begin{array}{l}\text { Cisplatin: } 80 \mathrm{mg} / \mathrm{m}^{2} \text {. } \\
\text { S:Surgery alone }\end{array}$ & \\
\hline $\begin{array}{l}\text { Burmeister, } \\
2005\end{array}$ & Australia & Oesophageal & (256)128 V.128 & CRT-S V.S & 65 & $\begin{array}{l}\text { CRT:35Gy } / 15 \mathrm{f} / 3 \mathrm{w} \text {; Cisplatin: } 80 \mathrm{mg} / \mathrm{m}^{2} \text {, } \\
\text { 5-Fu: } 800 \mathrm{mg} / \mathrm{m}^{2} \text {. }\end{array}$ & 8 \\
\hline & & $\operatorname{SCC}(61.7), \mathrm{AC}(37.1)$ & & & & S:Surgery alone & \\
\hline \multirow{3}{*}{$\begin{array}{l}\text { Klevebro, } \\
2016\end{array}$} & Sweden & Oesophageal(66), & (815)316 V.499 & CRT-S V.S & 48 V.60 & CRT:40Gy/2Gy/3w; & 8 \\
\hline & & GOJ(27.9) & & & & $\begin{array}{l}\text { AC:(Epirubicin:50mg/m²) } \\
\text { Cisplatin:(60)100mg/m², 5-Fu:(200)750mg/m². }\end{array}$ & \\
\hline & & SCC(26.4), AC(69.6) & & & & $\begin{array}{l}\text { SCC:Cisplatin: } 100 \mathrm{mg} / \mathrm{m}^{2}, 5-\mathrm{Fu}: 750 \mathrm{mg} / \mathrm{m}^{2} \text {. } \\
\text { S:Surgery alone }\end{array}$ & \\
\hline \multirow[t]{2}{*}{$\begin{array}{l}\text { Le Prise, } \\
1994\end{array}$} & France & Oesophageal & (86)41 V.45 & CRT-S V.S & NR & $\begin{array}{l}\text { CRT:40Gy/2Gy-20f/3w; Cisplatin: } 100 \mathrm{mg} / \mathrm{m}^{2} \text {, } \\
\text { 5-Fu:750mg/m². }\end{array}$ & 6 \\
\hline & & SCC & & & & S:Surgery alone & \\
\hline \multirow[t]{2}{*}{$\begin{array}{l}\text { Mariette, } \\
2014\end{array}$} & France & Oesophageal & (170)81 V.89 & CRT-S V.S & 93.6 & $\begin{array}{l}\text { CRT:45Gy/5Gy-25f/5w; Cisplatin:75mg/m², } \\
\text { 5-Fu: } 800 \mathrm{mg} / \mathrm{m}^{2} \text {. }\end{array}$ & 6 \\
\hline & & $\operatorname{SCC}(70.3), \mathrm{AC}(29.2)$ & & & & S:Surgery alone & \\
\hline \multirow[t]{2}{*}{$\begin{array}{l}\text { Natsugoe, } \\
2006\end{array}$} & Japan & Oesophageal & (43)20 V.23 & CRT-S V.S & 24 & $\begin{array}{l}\text { CRT:40Gy/2f/4w; Cisplatin:7mg/m², } \\
\text { 5-Fu:350mg/m² }\end{array}$ & 5 \\
\hline & & SCC & & & & S:Surgery alone & \\
\hline \multirow[t]{2}{*}{$\begin{array}{l}\text { Nygaard, } \\
1992\end{array}$} & Norway & Oesophageal & (103)53V.50 & CRT-S V.S & NR & $\begin{array}{l}\text { CRT:35Gy/1.75Gy-20f/4w; } \\
\text { Cisplatin:100mg/m², Bleomycin:50mg/m². }\end{array}$ & 5 \\
\hline & $\begin{array}{l}\text { Sweden/ } \\
\text { Finland }\end{array}$ & SCC & & & & S:Surgery alone & \\
\hline \multirow[t]{2}{*}{$\begin{array}{l}\text { Shapiro, } \\
2015\end{array}$} & Netherlands & Oesophageal(73.2), GOJ(24.0) & (366)178 V.188 & CRT-S V.S & 84.1 & $\begin{array}{l}\text { CRT: } 41.4 \mathrm{~Gy} / 1.8 \mathrm{~Gy}-23 \mathrm{f} / 5 \mathrm{w} \text {; } \\
\text { Paclitaxel:50mg } / \mathrm{m}^{2} \text {, carboplatin area under } \\
\text { curve }=2 \mathrm{mg} / \mathrm{ml} / \mathrm{min}\end{array}$ & 6 \\
\hline & & $\operatorname{SCC}(22.9), \mathrm{AC}(75.1)$ & & & & S:Surgery alone & \\
\hline \multirow[t]{2}{*}{ Tepper, 2008} & USA & Oesophageal(-), GOJ(-) & (56)30 V.26 & CRT-S V.S & 72 & $\begin{array}{l}\text { CRT:50.4Gy/1.8Gy/5.6w; } \\
\text { Cisplatin:100mg/m2, 5-Fu: } 1000 \mathrm{mg} / \mathrm{m}^{2}\end{array}$ & 8 \\
\hline & & $\begin{array}{l}\text { Stomach(-)/ } \\
\operatorname{SCC}(25.0), \operatorname{AC}(75.0)\end{array}$ & & & & S:Surgery alone & \\
\hline \multirow[t]{2}{*}{ Urba, 2001} & USA & Oesophageal(-), GOJ(-) & (100)50 V.50 & CRT-S V.S & 98.4 & $\begin{array}{l}\text { CRT:45Gy/1.5Gy/3w; Cisplatin:20mg/m², } \\
\text { 5-Fu:300mg/m². }\end{array}$ & 6 \\
\hline & & $\mathrm{SCC}(25.0), \mathrm{AC}(75.0)$ & & & & S:Surgery alone & \\
\hline \multirow[t]{2}{*}{$\begin{array}{l}\text { van Hagen, } \\
2012\end{array}$} & Netherlands & Oesophageal(73.2), GOJ(24.0) & (366)178 V.188 & CRT-S V.S & 45.4 & $\begin{array}{l}\text { CRT: } 41.4 \mathrm{~Gy} / 1.8 \mathrm{~Gy}-23 \mathrm{f} / 5 \mathrm{w} \text {; } \\
\text { Paclitaxel:50mg } / \mathrm{m}^{2} \text {, carboplatin area under } \\
\text { curve }=2 \mathrm{mg} / \mathrm{ml} / \mathrm{min}\end{array}$ & 7 \\
\hline & & $\operatorname{SCC}(22.9), \operatorname{AC}(75.1)$ & & & & S:Surgery alone & \\
\hline \multirow[t]{2}{*}{ Walsh, 1996} & Ireland & Oesophageal(65.5), GOJ(34.5) & (113)58 V.55 & CRT-S V.S & 10 & $\begin{array}{l}\text { CRT:40Gy/2.67Gy-20f/2w; } \\
\text { Cisplatin:75mg/m², 5-Fu:15mg/kg/d. }\end{array}$ & 7 \\
\hline & & $\mathrm{AC}$ & & & & S:Surgery alone & \\
\hline \multirow[t]{2}{*}{ Zhao, 2015} & China & GOJ & (76)36 V.40 & CRT-S V.S & NR & $\begin{array}{l}\text { CRT: } 45 \mathrm{~Gy} / 1.8 \mathrm{~Gy}-25 \mathrm{f} / 5 \mathrm{w} \\
\text { Capecitabine: } 1000 \mathrm{mg} / \mathrm{m}^{2} \\
\text { oxaliplatin: } 130 \mathrm{mg} / \mathrm{m}^{2}\end{array}$ & 7 \\
\hline & & $\mathrm{AC}$ & & & & S:Surgery alone & \\
\hline \multicolumn{8}{|c|}{ CRT-S V.CT-S } \\
\hline \multirow[t]{2}{*}{$\begin{array}{l}\text { Klevebro, } \\
2016\end{array}$} & Sweden & Oesophageal(82.9), GOJ(17.1) & (181)90 V.91 & $\begin{array}{l}\text { CRT-S } \\
\text { V.CT-S }\end{array}$ & NR & $\begin{array}{l}\text { CRT: } 40 \mathrm{~Gy} / 2 \mathrm{~Gy}-20 \mathrm{f} / 3 \mathrm{w} \text {; Cisplatin:100mg/m², } \\
\text { 5-Fu:750mg/m². }\end{array}$ & 8 \\
\hline & Norway & $\operatorname{SCC}(27.6), \mathrm{AC}(72.4)$ & & & & CT:Cisplatin:100mg/m², 5-Fu:750mg/m² & \\
\hline \multirow[t]{2}{*}{$\begin{array}{l}\text { Klevebro, } \\
2016\end{array}$} & Sweden & Oesophageal(63.3), GOJ(29.6) & (521)316 V.205 & $\begin{array}{l}\text { CRT-S } \\
\text { V.CT-S }\end{array}$ & 48 V.36 & CRT:40Gy/2Gy/3w; & 8 \\
\hline & & $\operatorname{SCC}(18.8), \operatorname{AC}(78.9)$ & & & & $\begin{array}{l}\text { AC:(Epirubicin:50mg/m²) } \\
\text { Cisplatin:(60)100mg/m², 5-Fu:(200)750mg/m². } \\
\text { SCC:Cisplatin:100mg/m², 5-Fu: } 750 \mathrm{mg} / \mathrm{m}^{2} \\
\text { CT: the same chemotherapy method as CRT }\end{array}$ & \\
\hline \multirow[t]{2}{*}{$\begin{array}{l}\text { Nygaard, } \\
1992\end{array}$} & Norway & Oesophageal & (109)53V.56 & $\begin{array}{l}\text { CRT-S } \\
\text { V.CT-S }\end{array}$ & NR & $\begin{array}{l}\text { CRT:35Gy/1.75Gy-20f/4w; } \\
\text { Cisplatin:100mg/m², Bleomycin:50mg/m². }\end{array}$ & 5 \\
\hline & $\begin{array}{l}\text { Sweden/ } \\
\text { Finland }\end{array}$ & SCC & & & & CT:Cisplatin:100mg/m², Bleomycin:50mg/m² & \\
\hline \multirow[t]{2}{*}{ Spicer, 2016} & American & Oesophageal(-), GOJ(-) & (214)100 V.114 & $\begin{array}{l}\text { CRT-S } \\
\text { V.CT-S }\end{array}$ & NR & $\begin{array}{l}\text { CRT:50.4Gy } / 3 w ; \text { Docetaxel: } 75 \mathrm{mg} / \mathrm{m}^{2} \\
\text { Cisplatin: } 75 \mathrm{mg} / \mathrm{m}^{2}, 5-\text { Fu: } 750 \mathrm{mg} / \mathrm{m}^{2}\end{array}$ & 5 \\
\hline & & $\mathrm{AC}$ & & & & $\begin{array}{l}\text { CT:Docetaxel:75mg/m², Cisplatin:75mg } / \mathrm{m}^{2} \text {, } \\
\text { 5-Fu:750mg } / \mathrm{m}^{2}\end{array}$ & \\
\hline \multirow[t]{2}{*}{ Stahl, 2009} & Germany & GOJ & (119)60 V.59 & $\begin{array}{l}\text { CRT-S } \\
\text { V.CT-S }\end{array}$ & 45.6 & $\begin{array}{l}\text { CRT:30Gy/2Gy15f/2w; Cisplatin:50mg/m², } \\
\text { 5-Fu:2000mg/m². }\end{array}$ & 7 \\
\hline & & $\mathrm{AC}$ & & & & CT:Cisplatin:50mg/m², 5-Fu:2000mg/m² & \\
\hline \multirow[t]{2}{*}{ Stahl, 2017} & Germany & GOJ & (119)60 V.59 & $\begin{array}{l}\text { CRT-S } \\
\text { V.CT-S }\end{array}$ & 126.5 & $\begin{array}{l}\text { CRT: } 40 \mathrm{~Gy} / 2 \mathrm{~Gy} 15 \mathrm{f} / 3 \mathrm{w} \text {; Cisplatin:50mg } / \mathrm{m}^{2} \text {, } \\
\text { etoposide: } 80 \mathrm{mg} / \mathrm{m}^{2}\end{array}$ & 7 \\
\hline & & $\mathrm{AC}$ & & & & CT:Cisplatin:50mg/m², 5-Fu:2000mg/m² & \\
\hline
\end{tabular}


NOS: Newcastle-Ottawa Quality Assessment Scale.

Table 2. Meta-analysis of overall survival rate for neoadjuvant chemoradiation (CRT) plus surgery (S) compared with neoadjuvant chemotherapy $(C T)$ plus surgery $(S)$ or surgery alone $(\mathrm{S})$

\begin{tabular}{|c|c|c|c|c|c|c|c|c|c|}
\hline \multirow{3}{*}{$\begin{array}{l}\text { Overall } \\
\text { survival }\end{array}$} & \multirow[t]{3}{*}{ No.of studies } & \multicolumn{4}{|c|}{ No.of patients } & \multirow{3}{*}{$\begin{array}{l}\text { Model for } \\
\text { meta-analysis }\end{array}$} & \multirow[t]{3}{*}{$R R(95 \% \mathrm{CI})$} & \multirow[t]{3}{*}{$I^{2}(\%)$} & \multirow{3}{*}{$\begin{array}{l}P \text { for } \\
\text { heterogeneity }\end{array}$} \\
\hline & & \multicolumn{2}{|c|}{ CRT+S } & \multicolumn{2}{|c|}{$\mathrm{CT}+\mathrm{S} / \mathrm{S}$} & & & & \\
\hline & & event & total & event & total & & & & \\
\hline $1 \mathrm{yr}$ & $14[6-9,11-12,14-21]$ & 916 & 1267 & 860 & 1288 & $\mathrm{~F}$ & $1.08(1.03,1.14)$ & $31 \%$ & 0.13 \\
\hline $2 \mathrm{yr}$ & $11[6-9,12,14-17,20-21]$ & 612 & 1146 & 516 & 1167 & $\mathrm{~F}$ & $1.21(1.12,1.32)$ & $29 \%$ & 0.17 \\
\hline $3 \mathrm{yr}$ & $14[6-9,11-12,14-21]$ & 482 & 1267 & 387 & 1288 & $\mathrm{R}$ & $1.31(1.09,1.58)$ & $56 \%$ & 0.005 \\
\hline $5 \mathrm{yr}$ & $12^{[7-8,12-21]}$ & 254 & 1103 & 189 & 1125 & $\mathrm{~F}$ & $1.38(1.17,1.62)$ & $27 \%$ & 0.18 \\
\hline pooled & & 2264 & 4783 & 1952 & 4868 & $\mathrm{~F}$ & $1.18(1.14,1.23)$ & $48 \%$ & 0.0001 \\
\hline
\end{tabular}

Abbreviations: CRT: chemoradiation; CT: chemotherapy; S: surgery; RR: relative risk; CI: confidence interval; F:fixed model; R: random model

Table 3. Meta-analysis of subgroup in different pathologies and tumor sizes for neoadjuvant chemoradiation (CRT) plus surgery (S) compared with neoadjuvant chemotherapy $(\mathrm{CT})$ plus surgery $(\mathrm{S})$ or surgery alone $(\mathrm{S})$

\begin{tabular}{|c|c|c|c|c|c|c|c|c|c|}
\hline \multirow[t]{3}{*}{ Overall survival } & \multirow[t]{3}{*}{ No.of studies } & \multicolumn{4}{|c|}{ No.of patients } & \multirow{3}{*}{$\begin{array}{l}\text { Model for } \\
\text { meta-analysis }\end{array}$} & \multirow[t]{3}{*}{$R R(95 \% \mathrm{CI})$} & \multirow[t]{3}{*}{$I^{2}(\%)$} & \multirow{3}{*}{$\begin{array}{l}P \text { for } \\
\text { heterogeneity }\end{array}$} \\
\hline & & \multicolumn{2}{|c|}{ CRT+S } & \multicolumn{2}{|c|}{$\mathrm{CT}+\mathrm{S} / \mathrm{S}$} & & & & \\
\hline & & event & total & event & total & & & & \\
\hline $\mathrm{ADC}$ & $9[8-10,14-17,20-21]$ & & & & & & & & \\
\hline $1 \mathrm{yr}$ & $9[8-10,14-17,20-21]$ & 681 & 913 & 738 & 1044 & $\mathrm{~F}$ & $1.06(1.01,1.12)$ & $10 \%$ & 0.35 \\
\hline $2 \mathrm{yr}$ & $9[8-10,14-17,20-21]$ & 471 & 913 & 484 & 1044 & $\mathrm{R}$ & $1.15(1.00,1.32)$ & $51 \%$ & 0.04 \\
\hline $3 \mathrm{yr}$ & $9[8-10,14-17,20-21]$ & 326 & 913 & 336 & 1044 & $\mathrm{R}$ & $1.18(0.94,1.49)$ & $65 \%$ & 0.004 \\
\hline $5 \mathrm{yr}$ & $8^{[8,10,14-17,20-21]}$ & 184 & 848 & 163 & 978 & $\mathrm{R}$ & $1.39(0.97,2.00)$ & $64 \%$ & 0.006 \\
\hline pooled & & 1662 & 3587 & 1721 & 4110 & $\mathrm{R}$ & $1.11(1.03,1.19)$ & $52 \%$ & 0.0002 \\
\hline SCC & $9[6-11,13-14,20]$ & & & & & & & & \\
\hline $1 \mathrm{yr}$ & $8^{[6-11,14,20]}$ & 320 & 468 & 328 & 531 & $\mathrm{~F}$ & $1.12(1.03,1.23)$ & $0 \%$ & 0.69 \\
\hline $2 \mathrm{yr}$ & $7[[6-10,14,20]$ & 211 & 427 & 186 & 486 & $\mathrm{~F}$ & $1.31(1.13,1.51)$ & $20 \%$ & 0.28 \\
\hline $3 \mathrm{yr}$ & $8[6-11,14,20]$ & 164 & 468 & 140 & 531 & $\mathrm{~F}$ & $1.34(1.11,1.61)$ & $0 \%$ & 0.49 \\
\hline $5 \mathrm{yr}$ & $6[7-9,13-14,20]$ & 81 & 369 & 72 & 434 & $\mathrm{~F}$ & $1.35(1.03,1.78)$ & $8 \%$ & 0.36 \\
\hline pooled & & 776 & 1732 & 726 & 1982 & $\mathrm{~F}$ & $1.23(1.15,1.33)$ & $7 \%$ & 0.36 \\
\hline Esophagus & $6[6-8,11-13]$ & & & & & & & & \\
\hline $1 \mathrm{yr}$ & $5[6-8,9,11-12]$ & 306 & 463 & 295 & 458 & $\mathrm{R}$ & $1.01(0.88,1.17)$ & $51 \%$ & 0.08 \\
\hline $2 \mathrm{yr}$ & $4[6-8,12]$ & 186 & 422 & 167 & 413 & $\mathrm{~F}$ & $1.09(0.94,1.28)$ & $17 \%$ & 0.31 \\
\hline $3 \mathrm{yr}$ & $5[6-8,9,11-12]$ & 138 & 463 & 129 & 458 & $\mathrm{~F}$ & $1.06(0.87,1.29)$ & $0 \%$ & 0.5 \\
\hline $5 \mathrm{yr}$ & $4[7-8,12-13]$ & 71 & 389 & 64 & 386 & $\mathrm{~F}$ & $1.12(0.84,1.50)$ & $0 \%$ & 0.76 \\
\hline pooled & & 701 & 1737 & 655 & 1715 & $\mathrm{~F}$ & $1.06(0.98,1.14)$ & $6 \%$ & 0.39 \\
\hline GOJ & $2^{[16-17]}$ & & & & & & & & \\
\hline $1 \mathrm{yr}$ & & 90 & 120 & 82 & 118 & $\mathrm{~F}$ & $1.08(0.92,1.26)$ & $0 \%$ & 1 \\
\hline $2 \mathrm{yr}$ & & 66 & 120 & 44 & 118 & $\mathrm{~F}$ & $1.47(1.11,1.96)$ & $0 \%$ & 0.75 \\
\hline $3 \mathrm{yr}$ & & 42 & 120 & 21 & 118 & $\mathrm{~F}$ & $1.97(1.25,3.08)$ & $0 \%$ & 0.52 \\
\hline $5 \mathrm{yr}$ & & 22 & 120 & 16 & 118 & $\mathrm{~F}$ & $1.35(0.78,2.35)$ & $0 \%$ & 0.38 \\
\hline pooled & & 220 & 480 & 163 & 472 & $\mathrm{~F}$ & $1.33(1.15,1.53)$ & $37 \%$ & 0.14 \\
\hline
\end{tabular}

Abbreviations: CRT: chemoradiation; CT: chemotherapy; S: surgery; RR: relative risk; CI: confidence interval; F: fixed model; R: random model; ADC: adenocarcinoma; SCC: squamous cell carcinoma; GOJ: gastro-oesophageal junction.

Table 4. Meta-analysis of DFS and PFS for neoadjuvant chemoradiation (CRT) plus surgery (S) compared with neoadjuvant chemotherapy $(\mathrm{CT})$ plus surgery $(\mathrm{S})$ or surgery alone $(\mathrm{S})$

\begin{tabular}{|c|c|c|c|c|c|c|c|c|c|}
\hline \multirow[t]{3}{*}{ Outcomes } & \multirow[t]{3}{*}{ No. of studies } & \multicolumn{4}{|c|}{ No. of patients } & \multirow{3}{*}{$\begin{array}{l}\text { Model for } \\
\text { meta-analysis }\end{array}$} & \multirow[t]{3}{*}{$R R(95 \% C I)$} & \multirow[t]{3}{*}{$I^{2}(\%)$} & \multirow{3}{*}{$\begin{array}{l}P \text { for } \\
\text { heterogeneity }\end{array}$} \\
\hline & & \multicolumn{2}{|c|}{ CRT+S } & \multicolumn{2}{|c|}{$\mathrm{CT}+\mathrm{S} / \mathrm{S}$} & & & & \\
\hline & & event & total & event & total & & & & \\
\hline DFS & $3[7,12,15]$ & & & & & & & & \\
\hline $1 \mathrm{yr}$ & & 209 & 341 & 189 & 349 & $\mathrm{~F}$ & $1.13(1.00,1.28)$ & $20 \%$ & 0.28 \\
\hline $2 \mathrm{yr}$ & & 139 & 341 & 131 & 349 & $\mathrm{~F}$ & $1.08(0.90,1.29)$ & $38 \%$ & 0.2 \\
\hline $3 \mathrm{yr}$ & & 92 & 341 & 94 & 349 & $\mathrm{~F}$ & $0.99(0.78,1.26)$ & $0 \%$ & 0.45 \\
\hline $5 \mathrm{yr}$ & & 47 & 341 & 53 & 349 & $\mathrm{~F}$ & $0.91(0.64,1.30)$ & $28 \%$ & 0.25 \\
\hline pooled & & 487 & 1364 & 467 & 1396 & $\mathrm{~F}$ & $1.06(0.97,1.17)$ & $0 \%$ & 0.45 \\
\hline PFS & $4[8,9,14,17]$ & & & & & & & & \\
\hline $1 \mathrm{yr}$ & $3[8,14,17]$ & 232 & 351 & 196 & 364 & $\mathrm{~F}$ & $1.23(1.09,1.39)$ & $0 \%$ & 0.46 \\
\hline $2 \mathrm{yr}$ & $3[8,14,17]$ & 179 & 351 & 134 & 364 & $\mathrm{~F}$ & $1.39(1.18,1.65)$ & $0 \%$ & 0.45 \\
\hline $3 \mathrm{yr}$ & $4[8,9,14,17]$ & 187 & 441 & 152 & 455 & $\mathrm{R}$ & $1.26(0.96,1.66)$ & $56 \%$ & 0.08 \\
\hline $5 \mathrm{yr}$ & $3[8,14,17]$ & 114 & 351 & 78 & 364 & $\mathrm{~F}$ & $1.53(1.20,1.95)$ & $0 \%$ & 0.71 \\
\hline pooled & & 712 & 1494 & 560 & 1547 & $\mathrm{~F}$ & $1.32(1.22,1.44)$ & $19 \%$ & 0.25 \\
\hline
\end{tabular}

Abbreviations: CRT: chemoradiation; CT: chemotherapy; S: surgery; RR: relative risk; CI: confidence interval; F: fixed model; R: random model; DFS: disease-free survival, PFS: progression-free survival; 
A

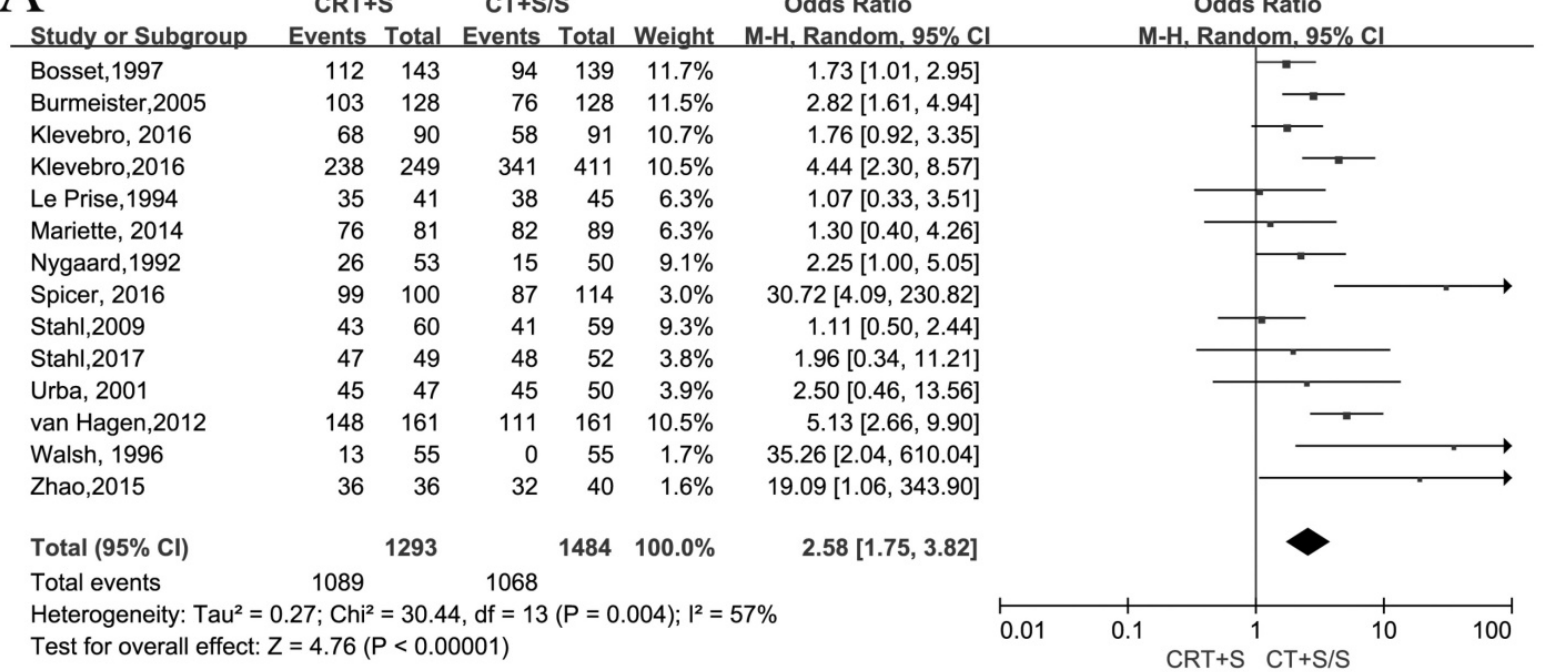

\begin{tabular}{|c|c|c|c|c|c|c|}
\hline \multirow[b]{2}{*}{ Study or Subgroup } & \multicolumn{2}{|c|}{ CRT+S } & \multicolumn{2}{|c|}{$\mathrm{CT}+\mathrm{S}$} & \multirow[b]{2}{*}{ Weight } & \multirow{2}{*}{$\begin{array}{c}\text { Odds Ratio } \\
\text { M-H. Fixed. } 95 \%\end{array}$} \\
\hline & Events & Total & Events & Total & & \\
\hline Klevebro, 2016 & 59 & 308 & 12 & 201 & $61.7 \%$ & $3.73[1.95,7.14]$ \\
\hline Klevebro,2016 & 22 & 90 & 7 & 91 & $27.6 \%$ & $3.88[1.56,9.63]$ \\
\hline Le Prise,1994 & 4 & 35 & 0 & 42 & $2.1 \%$ & $12.14[0.63,233.83]$ \\
\hline Stahl,2009 & 7 & 45 & 1 & 49 & $4.2 \%$ & $8.84[1.04,75.01]$ \\
\hline Stahl,2017 & 7 & 49 & 1 & 52 & $4.4 \%$ & $8.50[1.01,71.86]$ \\
\hline Total $(95 \% \mathrm{Cl})$ & & 527 & & 435 & $100.0 \%$ & $4.37[2.68,7.13]$ \\
\hline Total events & 99 & & 21 & & & \\
\hline \multicolumn{7}{|c|}{ Heterogeneity: $\mathrm{Ch}^{2}=1.54, \mathrm{df}=4(\mathrm{P}=0.82) ; \mathrm{I}^{2}=0 \%$} \\
\hline
\end{tabular}

\begin{tabular}{|c|c|c|c|c|c|c|}
\hline & CRT+ & & $\mathrm{CT}+\mathrm{S} /$ & & & Odds Ratio \\
\hline Study or Subgroup & Events & Total & Events & Total & Weight & M-H, Fixed, $95 \% \mathrm{C}$ \\
\hline Burmeister,2005 & 11 & 128 & 14 & 128 & $9.3 \%$ & $0.77[0.33,1.76]$ \\
\hline Klevebro,2016 & 13 & 90 & 15 & 91 & $9.2 \%$ & $0.86[0.38,1.92]$ \\
\hline Le Prise,1994 & 7 & 41 & 9 & 45 & $5.1 \%$ & $0.82[0.28,2.46]$ \\
\hline Mariette, 2014 & 15 & 98 & 28 & 96 & $17.3 \%$ & $0.44[0.22,0.89]$ \\
\hline Natsugoe,2006 & 1 & 20 & 1 & 23 & $0.6 \%$ & $1.16[0.07,19.80]$ \\
\hline Shapiro, 2015 & 39 & 178 & 72 & 188 & $39.5 \%$ & $0.45[0.29,0.72]$ \\
\hline Stahl,2017 & 4 & 45 & 8 & 48 & $5.1 \%$ & $0.49[0.14,1.75]$ \\
\hline Tepper,2008 & 1 & 30 & 3 & 26 & $2.2 \%$ & $0.26[0.03,2.71]$ \\
\hline Urba, 2001 & 8 & 50 & 19 & 50 & $11.5 \%$ & $0.31[0.12,0.80]$ \\
\hline Total $(95 \% \mathrm{CI})$ & & 680 & & 695 & $100.0 \%$ & $0.52[0.39,0.69]$ \\
\hline Total events & 99 & & 169 & & & \\
\hline Heterogeneity: Chi² & $2, \mathrm{df}=$ & $P=$ & 2); $\left.\right|^{2}=$ & & & \\
\hline
\end{tabular}

\section{D carss erass ous ante}

Study or Subgroup Events Total Events Total Weight M-H. Fixed. $95 \% \mathrm{CI}$

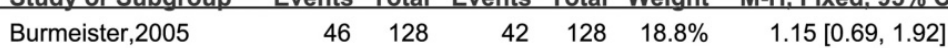
Le Prise 1994

Mariette, 2014

Natsugoe,2006

Shapiro, 2015

Stahl,2017

Tepper,2008

Urba, 2001

Total $(95 \% \mathrm{CI})$

$1.58[0.50,5.00]$

$0.70[0.37,1.34]$

$\begin{array}{rrrrr}22 & 98 & 28 & 96 & 15.3 \% \\ 8 & 20 & 4 & 23 & 1.6 \%\end{array}$

$3.17[0.78,12.85]$

$0.71[0.47,1.07]$

$0.52[0.22,1.24]$

$0.38[0.11,1.33]$

$1.08[0.49,2.38]$

$\begin{array}{rrrrr}13 & 45 & 21 & 48 & 10.1 \% \\ 5 & 30 & 9 & 26 & 5.6 \%\end{array}$

$\begin{array}{rrrrr}5 & 30 & 9 & 26 & 5.6 \% \\ 28 & 50 & 27 & 50 & 8.3 \%\end{array}$

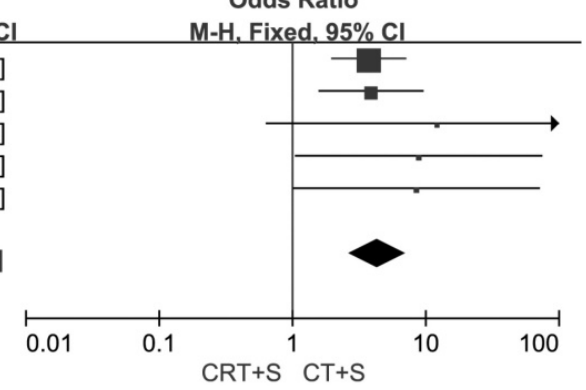

Odds Ratio

Total events

$590 \quad 604 \quad 100.0 \%$

$0.85[0.67,1.08]$

Heterogeneity: $\mathrm{Chi}^{2}=10.09, \mathrm{df}=7(\mathrm{P}=0.18) ; \mathrm{I}^{2}=31 \%$

Test for overall effect: $Z=1.33(P=0.19)$

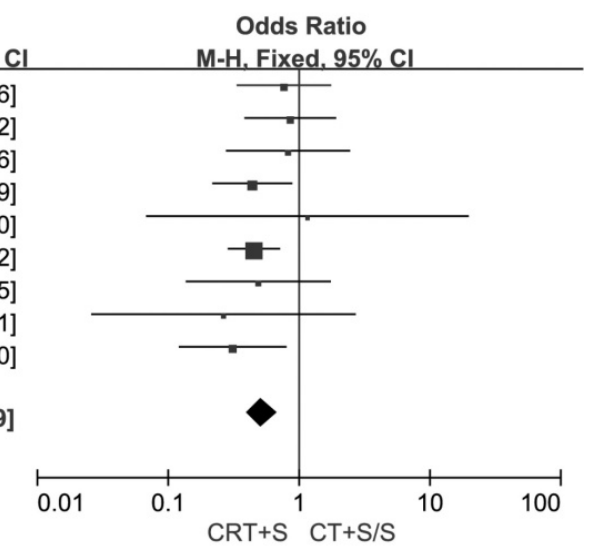

. Fixed, $95 \% \mathrm{Cl}$ 


\section{Treatment-related mortality and morbidity}

15 records [6-13, 15-21] evaluated the treatmentrelated mortality. Although the heterogeneity was not shown $(\mathrm{I} 2=29 \%, \mathrm{P}=0.14)$, the pooled OR of data from 15 records indicated no significant effect of neoadjuvant CRT plus surgery on resulting in treatmentrelated mortality (OR 1.27, 95\% CI 0.95-1.71, P=0.11) (Figure 3). The adverse events morbidity were also assessed. The meta-analysis data showed that higher incidences of grade3/4 bone marrow suppression and lymphatic fistula were observed in patients treated by neoadjuvant CRT plus surgery compared to neoadjuvant CT plus surgery or surgery alone. Neoadjuvant CRT plus surgery could increase the risk of cardiac complications and pulmonary embolism, even though there were no significant difference compared to neoadjuvant CT plus surgery or surgery alone(OR $1.09,95 \%$ CI $0.82-1.45, \mathrm{P}=0.57$; OR 2.06, 95\% CI 0.81-5.19, $\mathrm{P}=0.13)$.Oppositely, the patients conducted by neoadjuvant CRT plus surgery had higher incidences of bleed(OR 1.13, 95\% CI 0.36-3.59, $\mathrm{P}=0.84$ ), anastomotic fistula(OR 1.01, 95\% CI 0.76-1.35, $\mathrm{P}=0.94)$,
pulmonary(OR 2.06, 95\% CI 0.81-5.19, $\mathrm{P}=0.13$ ) and incision(OR 1.13, 95\% CI 0.56-2.29, $\mathrm{P}=0.72$ ) infection compared to neoadjuvant CT plus surgery or surgery alone, but no significant differences were shown between two arms. The pooled OR(95\%CI, P value) for adverse events morbidity was 1.14(0.99-1.32, 0.08 ),indicating that neoadjuvant CRT plus surgery did not increase the risk of adverse events morbidity(Table 5).

\section{Publication Bias and Sensitivity analysis}

Finally, the funnel plots were used to evaluate the publication bias of included records. As shown in Figure 4 , the plots were nearly symmetric. Hence, we didn't find significant publication bias in our meta-analysis. Sensitivity analysis (3 records which were evaluated as 5 scores removed from the procedure) was performed by excluding these 3 records. All indicated that the overall result was robust and the pooled RRs or ORs for outcomes were stable(data not shown).

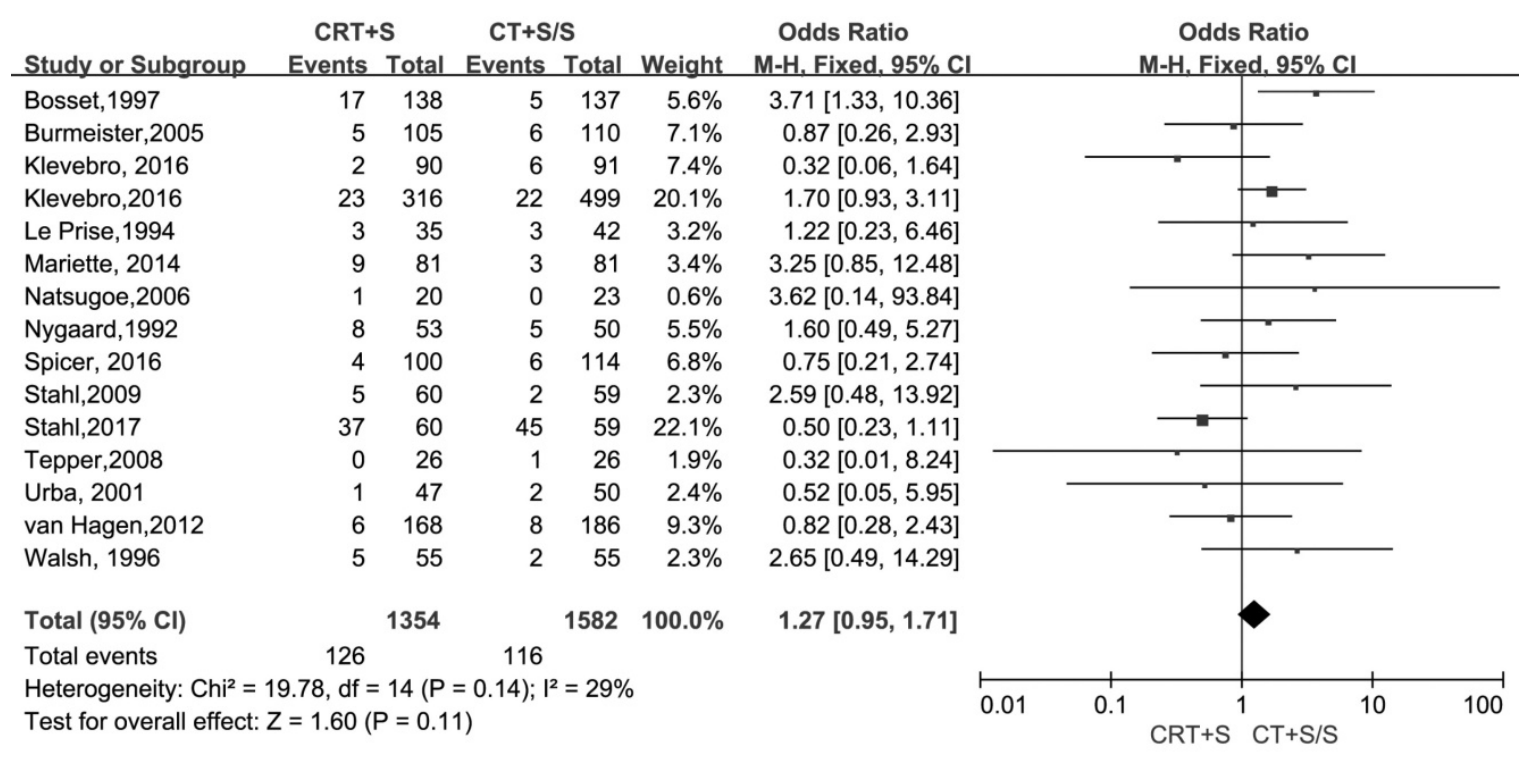

Figure 3. Treatment-related mortality in neoadjuvant chemoradiation (CRT) plus surgery $(\mathrm{S})$ compared with neoadjuvant chemotherapy $(\mathrm{CT})$ plus surgery $(\mathrm{S})$ or surgery alone $(S)$

Table 5. Meta-analysis of adverse events for neoadjuvant chemoradiation (CRT) plus surgery (S) compared with neoadjuvant chemotherapy (CT) plus surgery $(\mathrm{S})$ or surgery alone $(\mathrm{S})$

\begin{tabular}{|c|c|c|c|c|c|c|c|c|c|}
\hline \multirow[t]{3}{*}{ Adverse events } & \multirow[t]{3}{*}{ No. of studies } & \multicolumn{4}{|c|}{ No. of patients } & \multirow{3}{*}{$\begin{array}{l}\text { Model for } \\
\text { meta-analysis }\end{array}$} & \multirow[t]{3}{*}{ OR $(95 \% C I)$} & \multirow[t]{3}{*}{$I^{2}(\%)$} & \multirow{3}{*}{$\begin{array}{l}P \text { for } \\
\text { heterogeneity }\end{array}$} \\
\hline & & \multicolumn{2}{|c|}{ CRT+S } & \multicolumn{2}{|c|}{$\mathrm{CT}+\mathrm{S} / \mathrm{S}$} & & & & \\
\hline & & event & total & event & total & & & & \\
\hline Bone marrow suppression & $5[9,12,13,21-22]$ & 39 & 285 & 2 & 298 & F & $12.13(4.33,33.92)$ & $3 \%$ & 0.39 \\
\hline Anastomotic fistula & $11[6,8,10,11,13,15,18-22]$ & 100 & 978 & 114 & 1186 & $\mathrm{~F}$ & $1.01(0.76,1.35)$ & $0 \%$ & 0.77 \\
\hline Bleeding & $1[10]$ & 5 & 316 & 7 & 499 & $\mathrm{R}$ & $1.13(0.36,3.59)$ & - & - \\
\hline Abdominal infection & $1[10]$ & 4 & 316 & 5 & 499 & $\mathrm{R}$ & $1.27(0.34,4.75)$ & - & - \\
\hline Lymphatic fistula & $2^{[10,22]}$ & 13 & 352 & 7 & 539 & F & $2.83(1.14,6.99)$ & $0 \%$ & 0.9 \\
\hline Pneumonia & $8[6,8,10,11-13,15,21]$ & 117 & 791 & 153 & 1000 & $\mathrm{~F}$ & $0.82(0.62,1.08)$ & $0 \%$ & 0.67 \\
\hline Cardiovascular complications & $8[8-10,15,20-21]$ & 108 & 860 & 115 & 1073 & $\mathrm{~F}$ & $1.09(0.82,1.45)$ & $2 \%$ & 0.4 \\
\hline
\end{tabular}




\begin{tabular}{|c|c|c|c|c|c|c|c|c|c|}
\hline \multirow[t]{3}{*}{ Adverse events } & \multirow[t]{3}{*}{ No. of studies } & \multicolumn{4}{|c|}{ No. of patients } & \multirow{3}{*}{$\begin{array}{l}\text { Model for } \\
\text { meta-analysis }\end{array}$} & \multirow[t]{3}{*}{ OR(95\%CI) } & \multirow[t]{3}{*}{$I^{2}(\%)$} & \multirow{3}{*}{$\begin{array}{l}P \text { for } \\
\text { heterogeneity }\end{array}$} \\
\hline & & \multicolumn{2}{|c|}{ CRT+S } & \multicolumn{2}{|c|}{$\mathrm{CT}+\mathrm{S} / \mathrm{S}$} & & & & \\
\hline & & event & total & event & total & & & & \\
\hline Pulmonary & $3[10,11,18]$ & 10 & 375 & 7 & 565 & $\mathrm{~F}$ & $2.06(0.81,5.19)$ & $0 \%$ & 0.89 \\
\hline Incision infection & $2^{[15,18]}$ & 18 & 124 & 18 & 138 & $\mathrm{~F}$ & $1.13(0.56,2.29)$ & $0 \%$ & 0.87 \\
\hline pooled & & 544 & 5561 & 545 & 6758 & $\mathrm{~F}$ & $1.14(0.99,1.32)$ & $17 \%$ & 0.18 \\
\hline
\end{tabular}

Abbreviations: CRT: chemoradiation; CT: chemotherapy; S:surgery;OR: odd ratio; CI: confidence interval; F: fixed model; R: random model;"-": not applicable
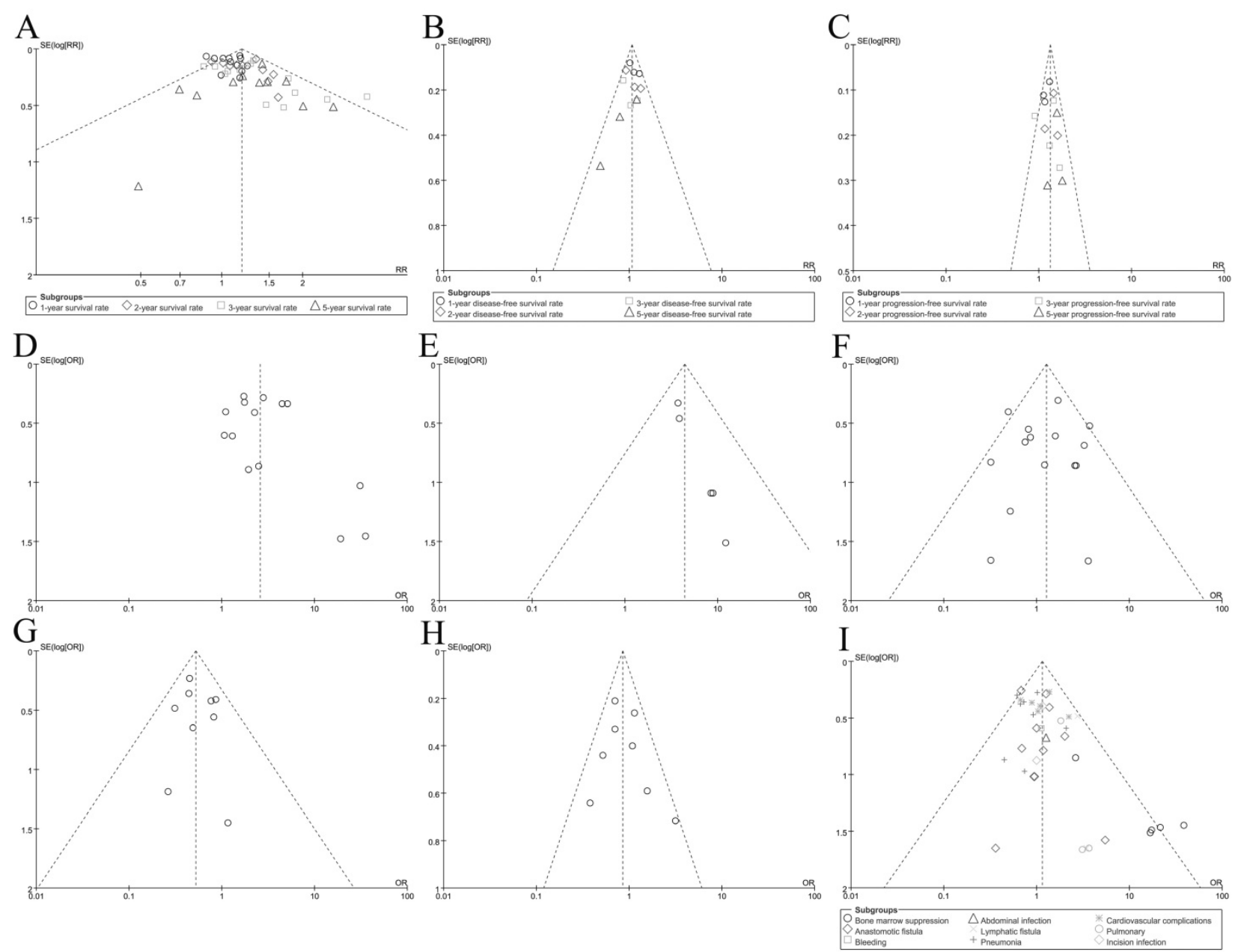

Figure 4. Funnel plot for outcomes; A: Overall survival(OS); B: disease-free survival(DFS); C: Progression-free survival(PFS); D: R0 resection rate; E: pCR rate; F: Treatment-related mortality; G: Local recurrence rate; $\mathrm{H}$ : Distant metastasis rate; I: Adverse event morbidity

\section{Discussion}

Gastroesophageal malignancies included distal oesophagus, proximal GOJ and distal gastric cancer (GC) [23].Both locally advanced carcinoma of the oesophagus and GOJ were related to poor prognosis due to the lack of effective treatment strategies [24]. Frequently cited multicenter trials such as MRC-OEO2, MAGIC and the FNLCC/FNDD trials on efficacy of a preoperative CT promoted the use of neoadjuvant CT in oesophagus or GOJ tumor, because of the significant survival benefit from neoadjuvant CT. Neoadjuvant CRT has had abundant potential advantages reported in recent decades. In contrast to neoadjuvant $\mathrm{CT}$, most neoadjuvant $\mathrm{CRT}$ studies and previous meta-analyses have consistently shown PCR rates of 15 to $25 \%$ and increased the chances of $\mathrm{R} 0$ resecability to reduce the incidence of local relapse. However, those trials of neoadjuvant CRT have only shown a trend towards improved survival compared with neoadjuvant CT or surgery alone, even although limited exciting research achievements were sill announced $[14,20]$. The discrepancies that whether neoadjuvant CRT improved the survival in the oesophagus or GOJ and the effect of the underlying histological subtype in determining the response to neoadjuvant CRT remains uncertain.

Our meta-analysis were based on 17 records related to the effect on patients with cancer of oesophagus or GOJ by comparing neoadjuvant CRT plus surgery to neoadjuvant CT plus surgery or 
surgery alone. A total number of patients in article was 4095 . The meta-analysis of data from 17 records indicated that a significant advantage on OS was observed in those patients with neoadjuvant CRT. The effect of neoadjuvant CRT plus surgery on survival was associated with a significant increase in 1-, 2-, 3and 5-year survival when compared to neoadjuvant CT plus surgery or surgery alone. A more accurate and systematic network-meta analysis also reported that neoadjuvant CRT improved overall survival when compared to all other treatments including surgery alone (HR 0.75, 95\% CR 0.67-0.85), neoadjuvant CT (HR 0.83,95\% CR $0.70-0.96$ ) [36]. However, the data above had been mixed by including different histology, tumor sizes and treatment strategies and the network-meta analysis didn't conduct a subgroup based on histology and tumor sizes. Meanwhile, it was believed that neoadjuvant CRT should be the treatment of choice for squamous cell carcinoma and CT may suffice for adenocarcinoma on the understanding that squamous cell carcinoma tumours are locally infiltrative, whereas adenocarcinoma primarily recurs distally with metastatic progression[25]. Thus, we conducted a subgroup analysis based on different histology and tumor sizes to estimate the effect for combined CRT. Unfortunately, the data from 9 records related to adenocarcinoma didn't show a survival benefit in comparing neoadjuvant CRT plus surgery to neoadjuvant CT plus surgery or surgery alone. Remarkably, the addition of radiotherapy for patients with adenocarcinoma seemingly improved 1- (RR 1.06, 95\% CI 1.01-1.12, $\mathrm{P}=0.03$ ) and 2-year survival (RR 1.15 , 95\% CI $1.00-1.32, \mathrm{P}=0.05)$, but the levels of statistical significance were very low and only a trend towards increasing survival was observed. Effects on OS in adenocarcinoma group were the same to the results from the recent meta-analysis of 1756 patients from 12 RCTs. The adenocarcinoma patients didn't benefit from neoadjuvant CRT(HR 0.72, 95\% CI 0.48-1.04)[37].Nevertheless, the effect of neoadjuvant CRT in small cell carcinoma group was more obvious in improving survival than that in adenocarcinoma group and significant differences in statistics were also detected. In terms of subgroup analysis by tumor sizes, patients with tumors in esophagus didn't benefit from neoadjuvant CRT, whereas those with tumors happened to GOJ appeared to obtain survival benefits. Basing on the understanding that esophageal cancer were classified as squamous cell carcinomas or adenocarcinomas, which squamous cell carcinomas were often found in esophagus and adenocarcinomas were happened to GOJ, these data above seemed to be contradictory. Obviously, the reasons for above opposite results might due to mix included SCC and
ADC in esophagus subgroup. We also found that only two records related to GOJ were included to evaluate effect of neoadjuvant CRT for patients with tumors in GOJ. As the famous results of van Hagen's and Shapiro' studies showed, the improvement on survival was both embodied in squamous cell carcinomas and adenocarcinomas, which was more prominent in patients with squamous cell carcinomas $[14,20]$. Similarly, a meta-analysis data from 9 randomized trials were pooled (1210 patients) and showed that overall survival was significantly increased at 2 years and 5 years $(P=0.002)$ with an absolute increase in survival of $7 \%$ in included exclusively squamous cell cancers trials[3]. At ASCO 2010 meeting, another important cross study from van der Gast was presented, also indicating that the patients benefited significantly from the neoadjuvant CRT in terms of a prolonged survival rate particularly in the small group of squamous cell carcinomas rather than the larger group of adenocarcinomas, which only showed a trend[26].This 'weak' effect for OS in adenocarcinomas histological type during neoadjuvant CRT might be caused by the diagnosis of adenocarcinoma of the distal esophagus (Barrett's carcinoma), which usually occurs in earlier stages compared to squamous cell carcinoma[24]. Thus, the above results suggested that the patients suffered from esophagus squamous cell carcinomas obviously benefited from neoadjuvant CRT.

No matter which histopathologic subtypes was suitable for neoadjuvant CRT, the effect of neoadjuvant CRT for DFS and PFS was coincident. No significant DFS benefit was observed in neoadjuvant CRT plus surgery group, although the trends improved 1-(RR 1.13, 95\% CI 1.00- 1.28, P=0.05) and 2-year (RR 1.08, 95\% CI 0.90-1.29, P=0.39) DFS were found by us. Surprisingly, our data indicated that the addition of pre-operation radiotherapy significantly postponed the disease progression time. The pooled RR (95\%CI, P value) for PFS was 1.30(1.19-1.43, $\mathrm{P}<0.00001)$. The increase for PFS by feat of neoadjuvant CRT may be mainly due to improving R0 resection and 'local control'. Consistent with results from most included RCTs and meta-analysis, our results also indicated that neoadjuvant CRT plus surgery increased $\mathrm{R} 0$ resection rate. The patients with neoadjuvant CRT plus surgery had higher percentage of pCR rate. At present, primary cause for debatable using of preoperative CRT was that the risk of disease progression during preoperative treatment. Obviously, our data pointed out that the patients treated by neoadjuvant CRT plus surgery had a lower incidence of local recurrence (OR 0.60, 95\% CI 0.48-0.75, $\mathrm{P}<0.00001)$. These results above demonstrated the reason that patients with neoadjuvant CRT had 
long-time PFS outcomes.

Another limiting application of neoadjuvant CRT reason was that a potential increase in the rate of adverse events after radical resection. While downsizing the tumor, controlling latent lymphatic and hematological micrometastasis, radiation also made tissues fragile, edematous or hemorrhagic, increasing possibility of anastomotic leakage, bleeding so much as therapy-related fateful complications [27]. However, we didn't notice that the neoadjuvant CRT plus surgery strategy increase treatment-related mortality. The pooled OR $(95 \% \mathrm{CI}, \mathrm{P}$ value) was $1.27(0.95-1.71,0.11)$. We also analyzed the incidence rate of adverse event morbidity, which was so called 'a nightmare for surgeon'. Once these adverse events arised, it would seriously affected survival and prognosis of patients with resectable esophagus or GOJ cancers. Our meta-analysis data indicated that neoadjuvant CRT plus surgery could increase incidences percentage of grade $3 / 4$ bone marrow suppression and lymphatic fistula. Although there was no significant difference to be observed on the risk of cardiac complications and pulmonary embolism, neoadjuvant CRT boosted the occurring possibility incidences rate of bleed(OR $1.13,95 \% \mathrm{CI}$ 0.36-3.59, $\mathrm{P}=0.84$ ), anastomotic fistula(OR 1.01, 95\% CI 0.76-1.35, $\mathrm{P}=0.94)$, pulmonary(OR 2.06, 95\% CI 0.81-5.19, $\mathrm{P}=0.13$ ) and incision(OR 1.13, 95\% CI $0.56-2.29, \mathrm{P}=0.72$ ) infection compared to neoadjuvant CT plus surgery or surgery alone, no significant differences were shown between two arms. The pooled $\mathrm{OR}(95 \% \mathrm{CI}, \mathrm{P}$ value) for adverse events morbidity was $1.14(0.99-1.32,0.08)$,indicating that neoadjuvant CRT plus surgery did not increase the risk of adverse events morbidity. Our results were also consistent with results of Skoropad V'study[28]. However, this seemingly safe strategy was not always reliable if once the radiation dose was superfluous. Radiation could alter the balance between collagen synthesis and matrix degradation. Rieff et al demonstrated that expressions of MMP2 and MMP9 in the normal esophagus were higher than patient in surgery alone group $[29,30]$. Thus, superfluous dose radiation was positively related to high risk of anastomotic fistula or infection.

Whether neoadjuvant CRT could provide additional survival advantage in potential patients, even in certain subgroups, was still waiting for more evidence due to the heterogeneity in therapeutic regimens and quality of trials included in published meta-analyses. Regarding the heterogeneity of scheme in the trials, it appeared difficult to draw any conclusions on dose-volume parameters and chemotherapy regimens. Data from included 17 RCT trails showed that the total dose of radiation ranged from $30 \mathrm{~Gy}$ to $50.4 \mathrm{~Gy}$ and the most scheme regimens of chemotherapy consisted of cisplatin and 5-Fu excluding expecting carboplatin/paclitaxed/oxaliplatin/ S-1[31]. Meanwhile, included these trials had a poor perform on recruitment, patient numbers, inadequate statistical power and so on. For instance, the RCT trail from Walsh et al lacked diagnostic computed tomography as an essential staging investigation and its poor statistics[21]. The small number was recruited by Urba et al[19]. Stahl et al' study closed early due to poor accrual[16]. The CALGB-9781 study intended to recruit 500 patients, but due to poor accrual and closed after only 56 patients[18]. In addition, most of included 17 trials were conducted in western countries and the actual efficacy and safety of neoadjuvant CRT in eastern countries were not understood.

The results of this meta-analysis were subject to several limitations: First, the relatively small number of studies and patients from GOJ cancers included. Only two records from the same medical center were included, which is likely to lead to influence meta-analysis of relevant results. Second, because of the lack of data from eastern countries, the conclusions were not global. Ethnic differences among different regions might have different radiochemotherapy sensitivity. Geny Piro et al indicated that different molecular pathways and genetic mutations from regions were involved in the carcinogenesis process of esophago-gastric adenocarcinoma [35].In addition, in order to evaluate the safety and efficacy of neoadjuvant CRT more comprehensively, we included as many literatures as possible conforming to the inclusion criteria. However, these RCT trials were extremely heterogeneous for histotypes, primary site, radiation dose and chemotherapy regimens, which were often mixing more chemosensitive adenocarcinomas with the squamous cell carcinoma subtype. Thus, those did not enable us to draw uniform conclusions.

Nowadays, some prospective clinical trials are carried out in Asia. Limited RCTs from Japan and South Korea are also reported and show that neoadjuvant CRT plays a vital role in offering advantage for survival[32].Above all, further more and cautious prospective RCTs should be designed and carried out. The TOPGEAR and RTOG 1010(NCT01196390) trial are ongoing and the final results are also exciting.

\section{Conclusion}

Our meta-analysis result demonstrated that neoadjuvant CRT plus surgery improved survival of patients with the oesophagus or GOJ cancers both in squamous cell carcinomas and adenocarcinomas. The 
patients with squamous cell carcinomas gained more survival advantage from neoadjuvant CRT. The addition of radiation was efficacy and safe in range. The data emerging from novel neoadjuvant CRT regimens is exciting, but needs further high-quality investigation based on inaccuracy from published prospective RCTs. We hope that our results could promote the continued development of innovative neoadjuvant CRT with novel methods and schedules of neoadjuvant CRT therapy.

\section{Abbreviations}

CRT: chemoradiation; CT: chemotherapy; GOJ: gastro-oesophageal junction; OR: odd ratio; CI: confidence interval; RCT: randomized controlled trial; ADC: adenocarcinoma; SCC: squamous cell carcinoma; OS: overall survival; DFS: disease-free survival, PFS: progression-free survival; ITT: intention-to-treat; pCR: pathological complete response rate.

\section{Supplementary Material}

Supplementary figures and tables. http://www.jcancer.org/v10p0192s1.pdf

\section{Acknowledgments}

This work was supported by grants from Natural Science Foundation of Liaoning Province (2015020 269)

\section{Competing Interests}

The authors have declared that no competing interest exists.

\section{References}

1. Daly JM, Karnell LH, Menck HR. National Cancer Data Base report on esophageal carcinoma. Cancer. 1996; 78: 1820-8

2. Blot WJ, Devesa SS, Kneller RW, Fraumeni JF, Jr. Rising incidence of adenocarcinoma of the esophagus and gastric cardia. Jama. 1991; 265: 1287-9.

3. Crehange G, Bonnetain F, Peignaux $\mathrm{K}$, Truc G, Blanchard N, Rat P, et al. Preoperative radiochemotherapy for resectable localised oesophageal cancer: a controversial strategy. Critical reviews in oncology/hematology. 2010; 75: 235-42

4. Mahadevan A, Carey D. The role of radiotherapy in the surgical management of gastrointestinal cancer. Surg Oncol. 1997; 6: 201-8.

5. Delaney JP, Bonsack ME, Felemovicius I. Lumenal route for intestinal radioprotection. Am J Surg. 1993; 166: 492-501.

6. Nygaard K, Hagen S, Hansen HS, Hatlevoll R, Hultborn R, Jakobsen A, et al. Pre-operative radiotherapy prolongs survival in operable esophageal carcinoma: a randomized, multicenter study of pre-operative radiotherapy and chemotherapy. The second Scandinavian trial in esophageal cancer. World J Surg. 1992; 16: 1104-9; discussion 10.

7. Bosset JF, Gignoux M, Triboulet JP, Tiret E, Mantion G, Elias D, et al. Chemoradiotherapy followed by surgery compared with surgery alone in squamous-cell cancer of the esophagus. The New England journal of medicine. 1997; 337: 161-7.

8. Burmeister BH, Smithers BM, Gebski V, Fitzgerald L, Simes RJ, Devitt P, et al Surgery alone versus chemoradiotherapy followed by surgery for resectable cancer of the oesophagus: a randomised controlled phase III trial. The Lancet Oncology. 2005; 6: 659-68.

9. Klevebro F, Alexandersson von Dobeln G, Wang N, Johnsen G, Jacobsen AB, Friesland S, et al. A randomized clinical trial of neoadjuvant chemotherapy versus neoadjuvant chemoradiotherapy for cancer of the oesophagus or gastro-oesophageal junction. Annals of oncology : official journal of the European Society for Medical Oncology / ESMO. 2016; 27: 660-7.

10. Klevebro F, Lindblad M, Johansson J, Lundell L, Nilsson M. Outcome of neoadjuvant therapies for cancer of the oesophagus or gastro-oesophageal junction based on a national data registry. The British journal of surgery. 2016; 103: 1864-73

11. Le Prise E, Etienne PL, Meunier B, Maddern G, Ben Hassel M, Gedouin D, et al. A randomized study of chemotherapy, radiation therapy, and surgery versus surgery for localized squamous cell carcinoma of the esophagus. Cancer. 1994; 73: 1779-84.

12. Mariette C, Dahan L, Mornex F, Maillard E, Thomas P-A, Meunier B, et al. Surgery Alone Versus Chemoradiotherapy Followed by Surgery for Stage I and II Esophageal Cancer: Final Analysis of Randomized Controlled Phase III Trial FFCD 9901. Journal of Clinical Oncology. 2014; 32: 2416-U201.

13. Natsugoe S, Okumura $H$, Matsumoto $M$, Uchikado $Y$, Setoyama $T$, Yokomakura N, et al. Randomized controlled study on preoperative chemoradiotherapy followed by surgery versus surgery alone for esophageal squamous cell cancer in a single institution. Diseases of the esophagus : official journal of the International Society for Diseases of the Esophagus. 2006; 19: 468-72.

14. Shapiro J, van Lanschot JJB, Hulshof $M$, van Hagen $P$, van Berge Henegouwen MI, Wijnhoven BPL, et al. Neoadjuvant chemoradiotherapy plus surgery versus surgery alone for oesophageal or junctional cancer (CROSS): long-term results of a randomised controlled trial. The Lancet Oncology. 2015; 16: 1090-8.

15. Spicer JD, Stiles BM, Sudarshan M, Correa AM, Ferri LE, Altorki NK, et al. Preoperative Chemoradiation Therapy Versus Chemotherapy in Patients Undergoing Modified En Bloc Esophagectomy for Locally Advanced Esophageal Adenocarcinoma: Is Radiotherapy Beneficial? The Annals of thoracic surgery. 2016; 101: 1262-9; discussion 969-70.

16. Stahl M, Walz MK, Stuschke M, Lehmann N, Meyer HJ, Riera-Knorrenschild J, et al. Phase III comparison of preoperative chemotherapy compared with chemoradiotherapy in patients with locally advanced adenocarcinoma of the esophagogastric junction. J Clin Oncol. 2009; 27: 851-6.

17. Stahl M, Walz MK, Riera-Knorrenschild J, Stuschke M, Sandermann A, Bitzer $\mathrm{M}$, et al. Preoperative chemotherapy versus chemoradiotherapy in locally advanced adenocarcinomas of the oesophagogastric junction (POET): Long-term results of a controlled randomised trial. Eur J Cancer. 2017; 81: 183-90.

18. Tepper J, Krasna MJ, Niedzwiecki D, Hollis D, Reed CE, Goldberg R, et al. Phase III trial of trimodality therapy with cisplatin, fluorouracil, radiotherapy, and surgery compared with surgery alone for esophageal cancer: CALGB 9781. J Clin Oncol. 2008; 26: 1086-92.

19. Urba SG, Orringer MB, Turrisi A, Iannettoni M, Forastiere A, Strawderman M. Randomized trial of preoperative chemoradiation versus surgery alone in patients with locoregional esophageal carcinoma. J Clin Oncol. 2001; 19: 305-13.

20. van Hagen $P$, Hulshof MCCM, van Lanschot JJB, Steyerberg EW, Henegouwen MIvB, Wijnhoven BPL, et al. Preoperative Chemoradiotherapy for Esophageal or Junctional Cancer. New England Journal of Medicine. 2012; 366: 2074-84

21. Walsh TN, Noonan N, Hollywood D, Kelly A, Keeling N, Hennessy TP. A comparison of multimodal therapy and surgery for esophageal adenocarcinoma. The New England journal of medicine. 1996; 335: 462-7.

22. Zhao Q, Li Y, Wang J, Zhang J, Qiao X, Tan B, et al. Concurrent Neoadjuvant Chemoradiotherapy for Siewert II and III Adenocarcinoma at Gastroesophageal Junction. The American journal of the medical sciences. 2015; 349: 472-6.

23. Sehdev A, Catenacci DV. Gastroesophageal cancer: focus on epidemiology, classification, and staging. Discovery medicine. 2013; 16: 103-11.

24. Matuschek C, Bolke E, Peiper M, Knoefel WT, Budach W, Erhardt A, et al. The role of neoadjuvant and adjuvant treatment for adenocarcinoma of the upper gastrointestinal tract. Eur J Med Res. 2011; 16: 265-74.

25. Siewert JR, Ott K. Are squamous and adenocarcinomas of the esophagus the same disease? Semin Radiat Oncol. 2007; 17: 38-44.

26. Knight G, Earle CC, Cosby R, Coburn N, Youssef Y, Malthaner R, et al. Neoadjuvant or adjuvant therapy for resectable gastric cancer: a systematic review and practice guideline for North America. Gastric cancer : official journal of the International Gastric Cancer Association and the Japanese Gastric Cancer Association. 2013; 16: 28-40.

27. Mezhir JJ, Tang LH, Coit DG. Neoadjuvant therapy of locally advanced gastric cancer. J Surg Oncol. 2010; 101: 305-14.

28. Skoropad V, Berdov B, Zagrebin V. Concentrated preoperative radiotherapy for resectable gastric cancer: 20-years follow-up of a randomized trial. J Surg Oncol. 2002; 80: 72-8.

29. Rieff EA, Hendriks T, Rutten HJ, Nieuwenhuijzen GA, Gosens MJ, van den Brule AJ, et al. Neoadjuvant radiochemotherapy increases matrix metalloproteinase activity in healthy tissue in esophageal cancer patients. Annals of surgical oncology. 2009; 16: 1384-9.

30. Vande Walle C, Ceelen WP, Boterberg T, Vande Putte D, Van Nieuwenhove Y, Varin $\mathrm{O}$, et al. Anastomotic complications after Ivor Lewis esophagectomy in patients treated with neoadjuvant chemoradiation are related to radiation dose to the gastric fundus. International journal of radiation oncology, biology, physics. 2012; 82: e513-9.

31. Hingorani M, Crosby T, Maraveyas A, Dixit S, Bateman A, Roy R. Neoadjuvant chemoradiotherapy for resectable oesophageal and gastro-oesophageal junction cancer--do we need another randomised trial? Clinical oncology (Royal College of Radiologists (Great Britain)). 2011; 23: 696-705. 
32. Hong YS, Lee JL, Park JH, Kim JH, Yoon SN, Lim SB, et al. Phase I study of preoperative chemoradiation with s-1 and oxaliplatin in patients with locally advanced resectable rectal cancer. International journal of radiation oncology, biology, physics. 2011; 79: 684-9.

33. Tomasello G, Petrelli F, Ghidini M, Pezzica E, Passalacqua R, Steccanella F, et al. Tumor regression grade and survival after neoadjuvant treatment in gastro-esophageal cancer: A meta-analysis of 17 published studies. Eur J Surg Oncol. 2017; 43: 1607-16.

34. Kumagai K, Rouvelas I, Tsai JA, Mariosa D, Lind PA, Lindblad M, et al. Survival benefit and additional value of preoperative chemoradiotherapy in resectable gastric and gastro-oesophageal junction cancer: a direct and adjusted indirect comparison meta-analysis. Eur J Surg Oncol. 2015; 41: 282-94.

35. Piro G, Carbone C, Santoro R, Tortora G, Melisi D. Predictive biomarkers for the treatment of resectable esophageal and esophago-gastric junction adenocarcinoma: from hypothesis generation to clinical validation. Expert review of molecular diagnostics. 2018; 18: 357-70.

36. Chan KKW, Saluja R, Delos Santos K, Lien K, Shah K, Cramarossa G, et al. Neoadjuvant treatments for locally advanced, resectable esophageal cancer: A network meta-analysis. Int J Cancer. 2018; 143: 430-7.

37. Liu B, Bo Y, Wang K, Liu Y, Tang X, Zhao Y, et al. Concurrent neoadjuvant chemoradiotherapy could improve survival outcomes for patients with esophageal cancer: a meta-analysis based on random clinical trials. Oncotarget. 2017; 8: 20410-7. 